\title{
Estrategias bioclimáticas para el diseño de edificios vinícolas
}

\section{Bioclimatic strategies for wineries design}

\author{
JIMÉNEZ-LÓPEZ, Verónica†*, LUNA-LEÓN, Anibal, BENNI, Stefano y BOJÓRQUEZ-MORALES, \\ Gonzalo
}

Universidad Xochicalco, Facultad de Arquitectura, Campus Mexicali, Baja California, México.

ID 1er Autor: Verónica, Jiménez-López / ORC ID: 0000-0003-1564-1479, CVU CONACYT ID: 399944.

ID $1^{\text {er }}$ Coautor: Anibal, Luna-León / ORC ID: 0000-0003-3480-0607, CVU CONACYT ID: 79641

ID $2^{\text {do }}$ Coautor: Stefano, Benni / ORC ID: 0000-0002-1425-172X

ID $3^{\text {er }}$ Coautor: Gonzalo, Bojórquez-Morales / ORC ID: 0000-0001-9303-9278, Researcher ID Thomson: C-8687-2018, CVU CONACYT ID: 79645, SNI CONACYT ID: 45005

DOI: $10.35429 /$ JSI.2020.15.4.7.18

Recibido Julio 10, 2020; Aceptado Diciembre 30, 2020

\section{Resumen}

La mayor cantidad de energía consumida en edificios vinícolas es utilizada para la refrigeración y humidificación interior, por esta razón el diseño correcto para lograr la eficiencia energética se ha convertido en un tema actual y de suma importancia para países productores del vino. La premisa del diseño de edificios vinícolas que requieren menor cantidad o nula energía para lograr condiciones controladas del ambiente higrotérmico interior para producción y envejecimiento del vino, permitió formular el objetivo del presente trabajo, que fue evaluar seis modelos de edificios vinícolas con estrategias bioclimáticas ubicados en el Valle de Guadalupe, Baja California a partir de datos de desempeño térmico (temperatura ambiente y humedad relativa interior) y consumo de energía (kWh y grados-hora), obtenidos por medio de simulación térmica dinámica. Se realizó la caracterización de la zona de estudio y con base en la revisión de trabajos diversos se definieron rangos óptimos de temperatura para el envejecimiento y producción de vino. Se diseñó un modelo base de edificio vinícola al que se le aplicaron estrategias bioclimáticas. Los resultados obtenidos permitieron sugerir cuales son las mejores opciones de diseño bioclimático para este tipo de edificios.

Edificios vinícolas, Diseño bioclimático, Eficiencia energética

\begin{abstract}
Greater amount of energy consumed in wineries is used for cooling and humidifying of the interior, for this reason the correct design of energy efficient wineries has become an important issue for winemaking countries. The purpose of the design of buildings that require less or no energy to achieve controlled conditions of the indoor hygrothermal environment for production and aging of wine, allowed to formulate the objective of this work, which was to evaluate six models of wineries with bioclimatic design located in El Valle de Guadalupe, Baja California from data on thermal performance (indoor temperature and relative humidity) and energy consumption (kWh and degrees-hour), obtained by dynamic thermal simulation. The zone of the study was characterized, based on the review of previous studies optimum temperature ranges were defined for aging and wine production. A basic model of a winemaking building was designed to which bioclimatic strategies were applied. The results obtained allowed to suggest the best bioclimatic design options for this type of buildings.
\end{abstract}

Citación: JIMÉNEZ-LÓPEZ, Verónica, LUNA-LEÓN, Anibal, BENNI, Stefano y BOJÓRQUEZ-MORALES, Gonzalo. Estrategias bioclimáticas para el diseño de edificios vinícolas. Revista de Innovación Sistemática. 2020. 4-15:7-18.

\footnotetext{
* Correspondencia al Autor (Correo Electrónico: veronicalopez@mxl.xochicalco.edu.mx)

$\uparrow$ Investigador contribuyendo como primer autor.
} 


\section{Introducción}

Los edificios vinícolas requieren de espacios para producción y envejecimiento del vino con control estricto de condiciones ambientales, esta premisa demanda edificios energéticamente eficientes y con un óptimo desempeño térmico. En el Valle de Guadalupe, en Baja California, el microclima de tipo mediterráneo es idóneo para el cultivo de variedades de vid, por lo cual, el $90 \%$ del vino mexicano se produce en esta región y es la zona enoturística de mayor afluencia económica en el país (Cavazos, 2012).

Sin embargo, la arquitectura y los materiales de construcción utilizados para los edificios vinícolas ubicados en la zona son diversos y la forma tiene mayor peso que la función, debido a las ventajas que representa un diseño atractivo para el turismo, con lo que se deja de lado o incluso se omiten las características constructivas necesarias para lograr un ambiente térmico interior adecuado para el vino.

Los pequeños productores de la región han implementado en sus edificios, algunos de manera empírica, estrategias pasivas de adecuación para disminuir costos por consumo de energía eléctrica para producción, envejecimiento y almacenamiento del vino, sin embargo, los requerimientos de temperatura ambiente y humedad relativa interior óptimos para producir vino, hacen necesario utilizar la mayor parte del día sistemas activos electromecánicos para el enfriamiento y la humidificación, lo que genera aumentos en el consumo energético.

El presente trabajo tiene como objetivo principal evaluar seis modelos con diseño bioclimático para edificios vinícolas a partir de la comparación de datos de desempeño térmico (temperatura ambiente y humedad relativa interior) y consumo de energía ( $\mathrm{kWh}$ y gradoshora), obtenidos por medio de simulación térmica dinámica, con las condiciones climáticas del Valle de Guadalupe.
El método empleado consideró: 1)Caracterización de la zona y del caso de estudio, 2)Determinación teórica de rangos óptimos de ambiente térmico para el vino, 3)Diseño de un modelo base de cava para los requerimientos de uso y de ambiente térmico de producción, envejecimiento y degustación del vino, 4)Simulación en estado dinámico de seis modelos de edificios con estrategias de diseño bioclimático, 5)Simulación en estado dinámico del microclima de la zona de estudio validado con datos de monitoreo y 6)Análisis de los resultados obtenidos con comparaciones en diferencias de porcentajes.

\section{Materiales y métodos}

\section{Zona y caso de estudio}

El Valle de Guadalupe se ubica entre los paralelos $30^{\circ}$ y $50^{\circ}$ latitud norte, dentro de las denominadas franjas geográficas del vino, que se consideran regiones con climas aptos para el crecimiento de vid, lo que le permite competir a la zona de estudio con los grandes países productores de vino a nivel mundial como Italia, España y Francia (Figura 1). Se ubica a más de 2 mil kilómetros de distancia de la Ciudad de México y a 100 kilómetros aproximadamente de la frontera suroeste de Estados Unidos, lo que facilita la afluencia del turismo internacional.

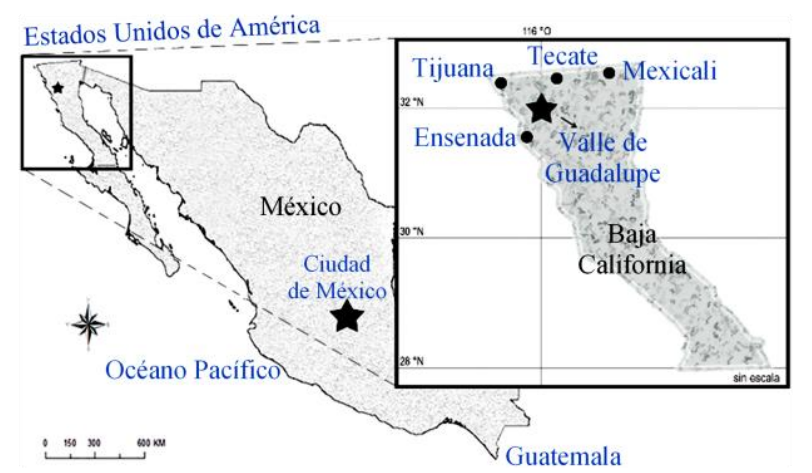

Figura 1 Macro y micro localización del caso de estudio Fuente: Elaboración propia

El Valle tiene una extensión territorial de $663.53 \mathrm{~km}^{2}$, dividida en tres delegaciones rurales: Francisco Zarco, San Antonio de las Minas y El Porvenir (Figura 2). El 77\% de los edificios vinícolas existentes en la zona de estudio pertenecen a pequeños productores quienes se caracterizan por utilizar procesos tradicionales de vinificación y tienen una producción menor a $900 \mathrm{hl}$ (10 000 cajas) de vino al año. 
El otro $23 \%$ está compuesto por productores medianos y de tipo industrial, estos últimos con maquinaria y tecnología que les permiten una producción anual mayor a $4500 \mathrm{hl}$. La investigación realizada se centra en los edificios vinícolas de tipo artesanal, es decir, en los pequeños productores.

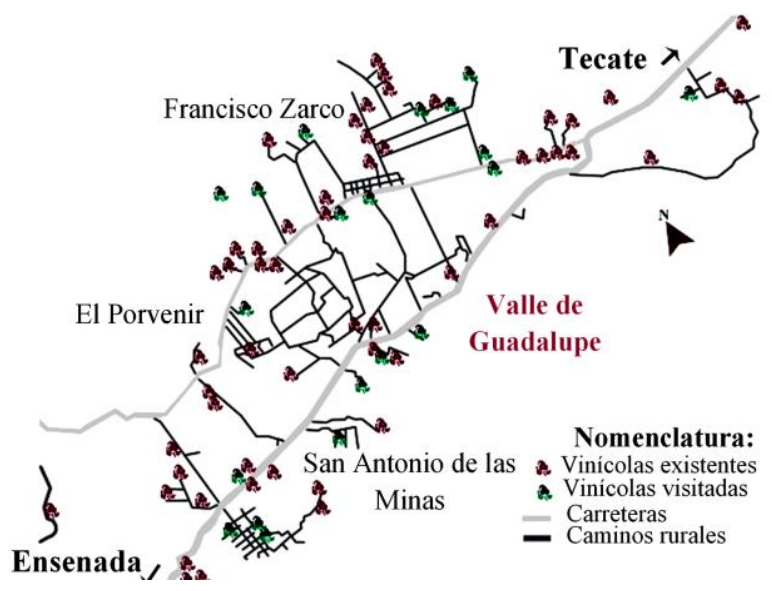

Figura 2 Ubicación de edificios vinícolas del Valle de Guadalupe hasta el año 2016

Fuente: Elaboración propia

A partir de recorridos en la zona de estudio, se caracterizó el $25 \%$ de las cavas existentes hasta el año 2016 (Figura 2), lo anterior, permitió definir que las áreas interiores con mayor requerimiento de control de temperatura ambiente y humedad relativa son la de producción y envejecimiento del vino, los sistemas constructivos más utilizados son el concreto armado y la estructura metálica, así como las tipologías constructivas de base y altura rectangular como las más repetidas en la zona.

\section{Modelos de cava de vino}

A partir de la revisión y análisis de los trabajos de Torreggiani, D., Benni, S., Corzani, V., Tassinari, P. y Galassi, S. (2011) y Tassinari, P., Torreggiani, D., Benni, S. y Corzani, V. (2012) se diseñó un modelo base de cava de vino. Para definir el diseño del modelo se analizó la relación de los factores siguientes: 1) Etapas de producción del vino, 2) Diseño del edificio, 3) Cantidad anual de vino producido y 4) Dimensiones (Altura, área y volumen).
Las dimensiones máximas obtenidas fueron altura de $4.50 \mathrm{~m}$, área total de 1472.65 $\mathrm{m}^{2}$ y volumen de $6626.93 \mathrm{~m}^{3}$. Las unidades espaciales y su distribución se definieron a partir de una matriz de relaciones y la forma en planta fue isodiamétrica, es decir, longitud y anchura con dimensiones iguales (Figura 3).

Al modelo base obtenido se integraron estrategias de diseño bioclimático que dieron como resultado la simulación de 11 edificios, seis modelos de cava de vino para el área de envejecimiento (UEt3) y cinco para el área de producción (UEt2), las cuales se mencionan en los párrafos siguientes.

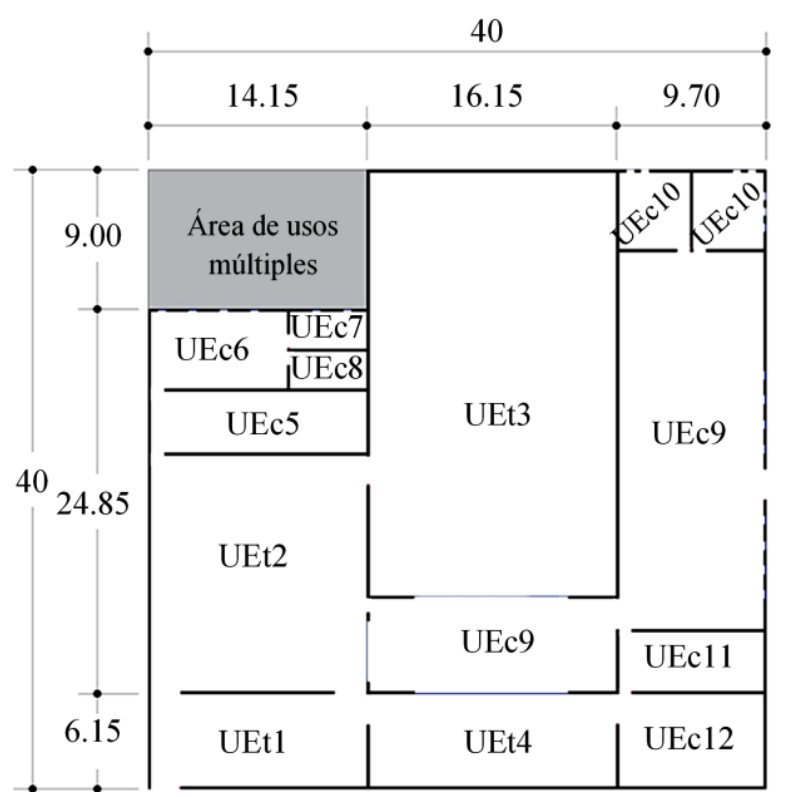

Figura 3 Solución de diseño para modelo de cava de vino artesanal. Planta de modelo base.

Simbología:

UEt1 - Ârea de recepción de uva: $84 \mathrm{~m}^{2}$

UEc5 - Área de guardado: $56 \mathrm{~m}^{2}$

UEc9 - Área de degustación: $96 \mathrm{~m}^{2}$

UEt2 - Área de producción (vinificación): $213.5 \mathrm{~m}^{2}$

UEc6 - Área de vestidor: $44.3 \mathrm{~m}^{2}$

UEc10 - Área de baño para visitantes: $23.1 \mathrm{~m}^{2}$

UEt3 - Área de envejecimiento y preservación de vino: $438.4 \mathrm{~m}^{2}$

UEc7 - Área de regaderas: $12.1 \mathrm{~m}^{2}$

UEc11 - Área de venta: $36.2 \mathrm{~m}^{2}$

UEt4 - Área de embotellado y empacado: $96 \mathrm{~m}^{2}$

UEc8 - Área de baño para trabajadores: $12.1 \mathrm{~m}^{2}$

UEc12 - Área de almacenaje de producto terminado: 56.4 $\mathrm{m}^{2}$

Fuente: Elaboración propia 
Para definir por orden de prioridad cuál elemento de la envolvente era el que requería la aplicación de alguna estrategia bioclimática, se realizaron simulaciones de prueba en el programa DesignBuilder con el modelo base en tres configuraciones constructivas: 1) Nivel natural del terreno, 2) Subterráneo y 3) Semienterrado (Figura 4).

Los resultados obtenidos mostraron que los techos en las tres configuraciones aportaban mayor carga térmica al edificio, seguido de los muros. Por lo anterior, se decidió aplicar a cada modelo de diseño del edificio estrategias bioclimáticas de masa térmica (techo verde, taludes de tierra en muros y muros subterráneos), sombreado (doble cubierta y muro sombreado) y aislamiento térmico (se utilizó el poliuretano por ser el aislante térmico de mayor uso en la región) como material de comparación.

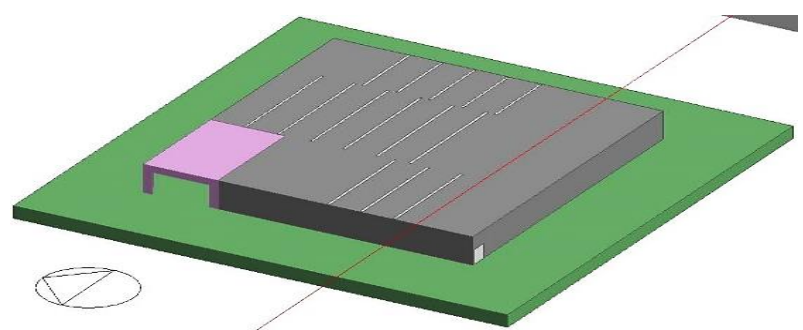

4a) Edificio base, al nivel natural del terreno

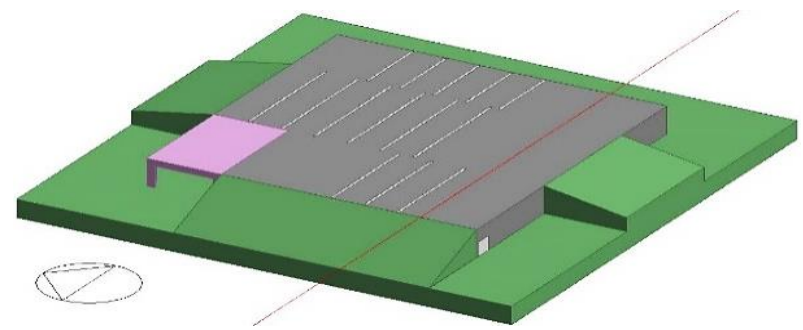

4b) Edificio semi-enterrado.

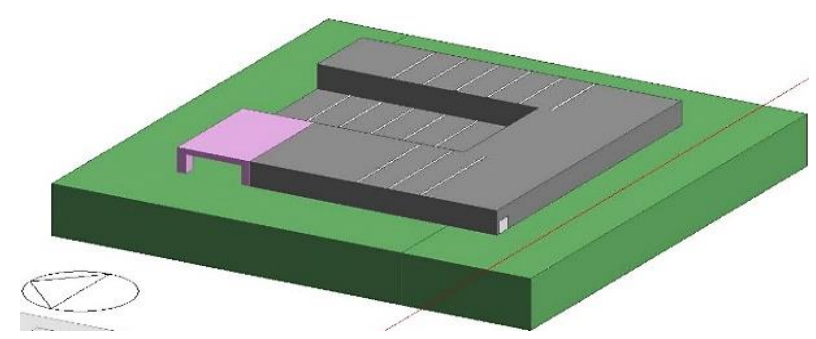

4c) Edificio subterráneo

Figura 4 Modelos de diseño para cava de vino. Isométricos en DesignBuilder

Fuente: Elaboración propia.

Las estrategias de diseño se aplicaron a los edificios propuestos según lo descrito en la Figura 5.
En el presente trabajo se muestran los resultados obtenidos para el área de envejecimiento, los resultados del área de producción se discutirán en artículos posteriores.

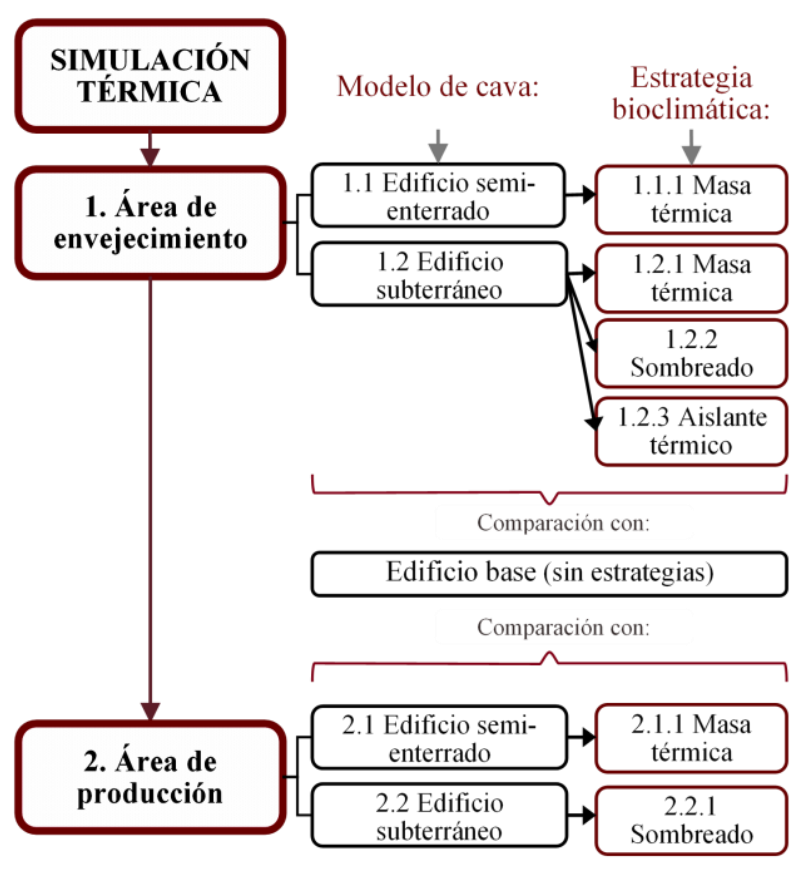

Figura 5 Variaciones de modelos de edificios vinícolas para simulación térmica

Fuente: Elaboración propia

\section{Simulación térmica}

La simulación térmica se realizó con el programa DesignBuilder® que utiliza la plataforma de EnergyPlus ${ }^{\circledR}$. Debido a la detallada proporción de datos que solicita este programa de simulación, a continuación, se menciona una lista de las consideraciones realizadas:

El edificio se dividió en 12 unidades espaciales según los requerimientos para transformación de la uva en vino (UEt) y su comercialización (UEc) ver Figura 2.

- Se determinaron la latitud y longitud del edificio, mediante un sistema de posición geográfica (GPS, Geographic Position System).

Se consideró como archivo meteorológico (tipo epw, por sus siglas en inglés que significan enegy plus weather file) el del ejido El Porvenir en el Valle de Guadalupe, este microclima se monitoreó durante un año con una estación meteorológica, lo que permitió la comparación de datos simulados con datos de monitoreo para validar el entorno de la simulación.

JIMÉNEZ-LÓPEZ, Verónica, LUNA-LEÓN, Anibal, BENNI, Stefano y BOJÓRQUEZ-MORALES, Gonzalo. Estrategias bioclimáticas para el diseño de edificios vinícolas. Revista de Innovación Sistemática. 2020 
- $\quad$ El aire acondicionado se consideró como desactivado (en simulaciones de prueba), para conocer el efecto de los materiales de la envolvente del edificio en la temperatura de bulbo seco y humedad relativa interior. Lo anterior, para definir que elemento de la envolvente en los modelos era el más débil al aportar mayor carga térmica.

Se realizaron programaciones de ocupación por usuarios, refrigeración, calefacción e iluminación para el análisis de cargas internas con base en observaciones de actividades de operación de las cavas de referencia.

Se tomaron en cuenta las sombras exteriores.

Se consideró la conductividad térmica del tipo de suelo para el Valle de Guadalupe como limo arcilloso.

Para el área de envejecimiento en el modelo subterráneo se consideró una profundidad de $4.5 \mathrm{~m}$ por debajo del nivel natural del terreno, que es igual a la altura de los muros restantes que no son subterráneos.

Se consideraron muros interiores, huecos de escaleras y la relación entre los espacios, así como la transferencia de energía entre ellos.

Se consideraron los coeficientes convectivos para muros, cubiertas y pisos.

En los modelos propuestos se tomó en cuenta el diseño estructural de losas y muros, así como su intercambio de energía con el ambiente interior.

Los edificios simulados incluyeron en su estructura vigas de acero para claros de más de $10 \mathrm{~m}$ de distancia.

Se tomaron en cuenta valores de aislamiento por ropa (clo), iluminación de los espacios (lux), requerimiento de aire fresco (1/s-person), de ventilación mecánica por área $\left(1 / \mathrm{s}-\mathrm{m}^{2}\right)$ y la masa térmica interior del área de envejecimiento (cantidad de barricas por $\mathrm{m}^{2}$ ).
- Se tomaron en cuenta valores de aislamiento por ropa (clo), iluminación de los espacios (lux), requerimiento de aire fresco (1/s-person), de ventilación mecánica por área $\left(1 / \mathrm{s}-\mathrm{m}^{2}\right)$ y la masa térmica interior del área de envejecimiento (cantidad de barricas por $\mathrm{m}^{2}$ ).

- Los parámetros para simulación del techo verde se definieron a partir de los trabajos de Ziogou, I., Apostolos, M., Vasiliki, V. y Theodoros, Z. (2017) y Sailor, D.J. (2008).

Se establecieron promedios de rangos teóricos, con base en autores varios, óptimos para el envejecimiento $\left(10^{\circ} \mathrm{C}\right.$ a 15 $\left.{ }^{\circ} \mathrm{C}\right)$ y la producción del vino $\left(15^{\circ} \mathrm{C}\right.$ a 25 $\left.{ }^{\circ} \mathrm{C}\right)$, ver Tabla 1.

\begin{tabular}{|l|l|}
\hline \multicolumn{1}{|c|}{ Autor } & \multicolumn{1}{c|}{$\begin{array}{c}\text { Temperatura de bulbo } \\
\text { seco }\left({ }^{\circ} \mathbf{C}\right)\end{array}$} \\
\hline Troost (1953) & $9-15$ para vinos tintos \\
\hline Boulton et al. (1995) & $5-15$ \\
\hline Hidalgo (2003) & $9-12$ \\
\hline $\begin{array}{l}\text { Mazarrón y Cañas } \\
\text { 2008) }\end{array}$ & $\leq 18$ \\
\hline Steiner (2010) & $13-20$ \\
\hline $\begin{array}{l}\text { Mazarrón, Cid - } \\
\text { Falceto y Cañas (2012) }\end{array}$ & $8-15$ \\
\hline $\begin{array}{l}\text { Bondiac, citado en } \\
\text { Barbaresi, et al. (2014) }\end{array}$ & $10-12$ \\
\hline $\begin{array}{l}\text { Marescalchi, citado en } \\
\text { Barbaresi, et al. (2014) }\end{array}$ & $15-20$ \\
\hline $\begin{array}{l}\text { Vogt, citado en } \\
\text { Barbaresi, et al. (2014) }\end{array}$ & $8-12$ para vinos blancos y \\
\hline Marrara et al. (2014) & $12-16$ \\
\hline $\begin{array}{l}\text { Considine y Frankish } \\
\text { (2014) }\end{array}$ & $\leq 20$ para vinos tintos y $<$ \\
\hline
\end{tabular}

Tabla 1 Temperatura ambiente interior óptima para el área de envejecimiento en una cava de vino Fuente: Elaboración propia

La validación de la simulación térmica se realizó con un método empírico (San Isidro, 1998; Mateus, Pinto y Carrilho Da Graca, 2014), que consistió en un análisis estadístico comparativo de datos del entorno de simulación con datos obtenidos por monitoreo, se utilizó una cava de referencia, ubicada en el Ejido El Porvenir en el Valle de Guadalupe (Figura 6). Se comparó la temperatura de bulbo seco del periodo cálido, del mes de mayo al mes de septiembre y del periodo frío de diciembre a febrero, estos datos fueron los obtenidos con la simulación térmica en el programa DesignBuilder y con el monitoreo en sitio. 


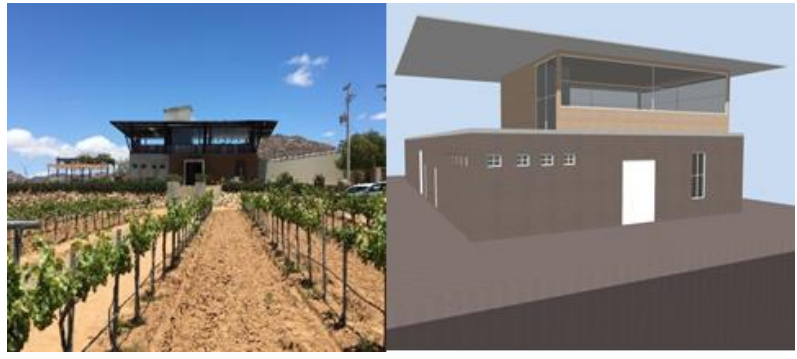

Figura 6 Izquierda: Cava de vino de referencia. Derecha: Cava de referencia en simulador

Fuente: Elaboración propia

En el periodo cálido se analizaron 3672 datos de temperatura de bulbo seco (mayoseptiembre). La comparación de los resultados de monitoreo con la simulación tuvo un error máximo positivo de $+4.4{ }^{\circ} \mathrm{C}$ y un máximo negativo de $-3.6{ }^{\circ} \mathrm{C}$, una apertura gráfica del error de $\pm 4{ }^{\circ} \mathrm{C}$ (Figura 7). Es necesario especificar que cuando los datos de simulación fueron menores a los datos de monitoreo el error fue considerado como positivo y cuando la simulación presentó datos mayores a los del monitoreo el error se consideró negativo.

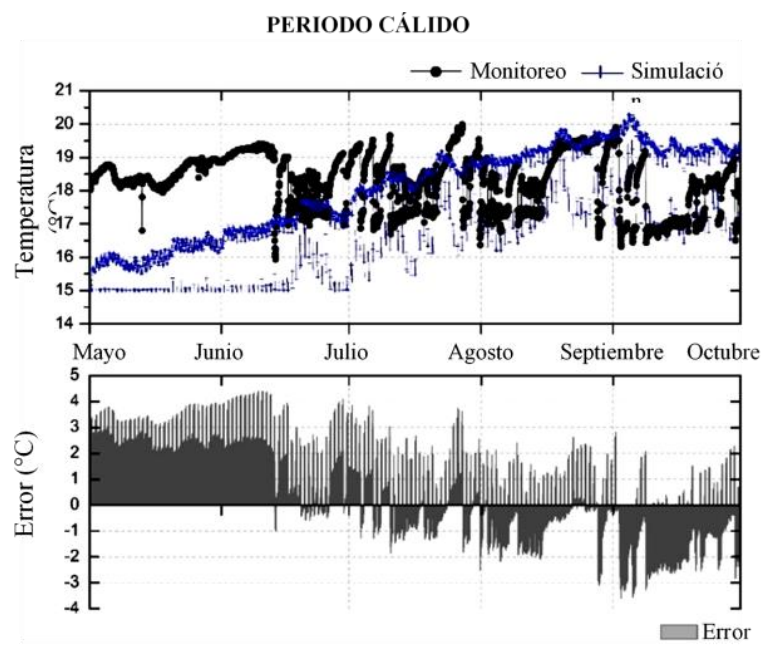

Figura 7 Validación de simulación térmica. Error entre datos de monitoreo y simulación en el periodo cálido Fuente: Elaboración propia

En el periodo frío se analizaron 2157 datos, se obtuvo un error máximo positivo de $+1.8^{\circ} \mathrm{C}$ y un error máximo negativo de $-1.7^{\circ} \mathrm{C}$, una apertura gráfica del error de $\pm 1.75^{\circ} \mathrm{C}$ como se muestra en la Figura 8.

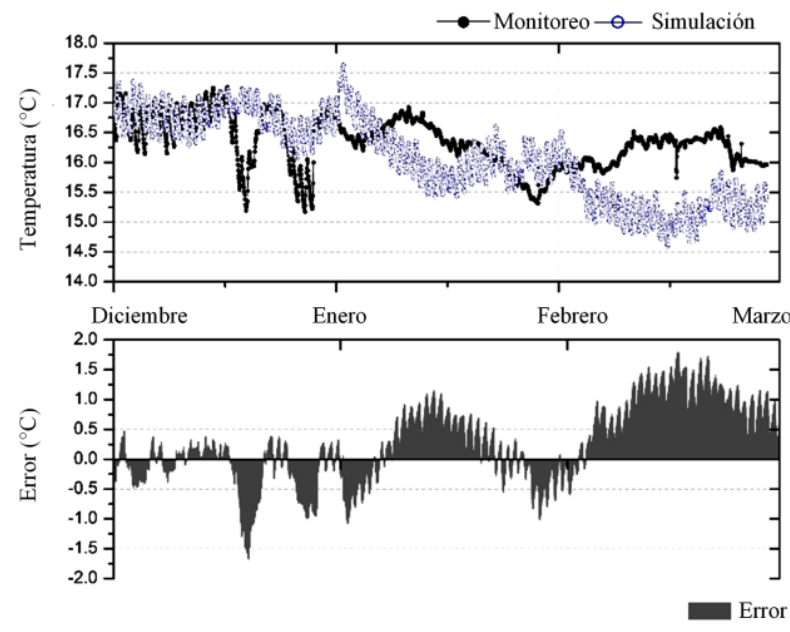

Figura 8 Validación de simulación térmica. Error entre datos de monitoreo y simulación en el periodo frío

Fuente: Elaboración propia

En los meses del periodo cálido la desviación absoluta media fue de $1.58{ }^{\circ} \mathrm{C}$ y el porcentaje de error absoluto medio fue de $8.7 \%$ entre la simulación y el monitoreo térmico, mientras que el coeficiente de determinación múltiple $\left(\mathrm{R}^{2}\right)$ fue de 0.04 , lo que mostró una relación directa entre los dos grupos de datos comparados. En el periodo frío la desviación absoluta media fue de $0.56^{\circ} \mathrm{C}$ y el porcentaje de error absoluto medio fue de $3.47 \%$, la $\mathrm{R}^{2}$ fue de 0.16 (Tabla 2).

\begin{tabular}{|l|c|c|}
\hline \multicolumn{1}{|c|}{ Indicador de validación } & \multicolumn{1}{c}{$\begin{array}{c}\text { Periodo } \\
\text { cálido }\end{array}$} & $\begin{array}{c}\text { Periodo } \\
\text { frío }\end{array}$ \\
\hline Error medio $\left({ }^{\circ} \mathrm{C}\right)$ & 0.54 & 0.26 \\
\hline $\begin{array}{l}\text { Desviación absoluta media } \\
\left({ }^{\circ} \mathrm{C}\right)\end{array}$ & 1.58 & 0.56 \\
\hline $\begin{array}{l}\text { Coeficiente de determinación } \\
\text { múltiple }\left(\mathrm{R}^{2}\right)\end{array}$ & 0.04 & 0.16 \\
\hline $\begin{array}{l}\text { Porcentaje de error absoluto } \\
\text { medio }(\%)\end{array}$ & 8.67 & 3.47 \\
\hline $\begin{array}{l}\text { Error medio en máximas } \\
\text { diarias }\left({ }^{\circ} \mathrm{C}\right)\end{array}$ & 0.50 & 0.10 \\
\hline $\begin{array}{l}\text { Desviación absoluta media } \\
\text { en máxima diaria }\left({ }^{\circ} \mathrm{C}\right)\end{array}$ & 1.29 & 0.48 \\
\hline $\begin{array}{l}\text { Coeficiente de determinación } \\
\text { múltiple en máxima diaria } \\
\left(\mathrm{R}^{2}\right)\end{array}$ & 0.002 & 0.27 \\
\hline $\begin{array}{l}\text { Porcentaje de error absoluto } \\
\text { medio en máxima diaria }(\%)\end{array}$ & 6.95 & 2.91 \\
\hline
\end{tabular}

Tabla 2 Validación de resultados de simulación térmica Fuente: Elaboración propia 
El error medio $\left({ }^{\circ} \mathrm{C}\right)$ obtenido, tanto para el periodo cálido como el periodo frío, se ubicó dentro de los rangos aceptables para validación empírica de simulaciones de edificios con EnergyPlus, de acuerdo con lo que mencionan Mateus, Pinto y Carrhilo Da Graca (2014), quienes mencionan errores de $4.3{ }^{\circ} \mathrm{C}$ a $7{ }^{\circ} \mathrm{C}$. También se realizó una comparación con los valores obtenidos de porcentaje de error absoluto medio (\%) y la simulación de los modelos propuestos entraron en la clasificación de excelente $(3.47 \%)$ en el periodo frío y muy bueno en el periodo cálido (8.67\%), según la clasificación de Ali y Abustan (2014).

\section{Resultados}

Periodo frío.

Este incluyó los meses de diciembre, enero y febrero y se analizaron: 1) Temperatura de bulbo seco interior $\left.\left({ }^{\circ} \mathrm{C}\right), 2\right)$ Grados por hora mensuales $\left({ }^{\circ} \mathrm{C}\right)$ fuera y dentro del rango higrotérmico óptimo para el vino y 3) Enfriamiento o calentamiento sensible requerido $(\mathrm{kWh})$.

1) Los datos obtenidos de temperatura de bulbo seco interior (TBS), mostraron que en promedio $61 \%$ de las horas la TBS se ubicó arriba del límite de $15^{\circ} \mathrm{C}$ y $39 \%$ de las horas de este periodo se ubicaron dentro del rango óptimo establecido para envejecimiento del vino de 10 ${ }^{\circ} \mathrm{C}$ a $15^{\circ} \mathrm{C}$. Lo anterior permitió definir que, para esta área de la cava, durante el periodo frío, no es necesario el uso de calefacción, pero sí se requiere enfriamiento electromecánico en todos los edificios propuestos para alcanzar los valores de temperatura del rango óptimo definido.

En cuanto al análisis de la oscilación de la temperatura interior, en el modelo con doble techo la cubierta del área de envejecimiento estuvo sombreada, pero en contacto con el aire del exterior, esto se reflejó en una diferencia entre la TBS máxima y la TBS mínima registrada de $5.25{ }^{\circ} \mathrm{C}$, en comparación con el edificio con techo verde con una oscilación de $3.61{ }^{\circ} \mathrm{C}$. El edificio con techo verde presentó valores de temperatura interior por encima del rango óptimo, pero fue uno de los modelos más estables al tener menor diferencia entre la TBS mínima y TBS máxima horaria (Tabla 3).

\begin{tabular}{|l|c|r|r|r|r|}
\hline $\begin{array}{l}\text { Modelo de } \\
\text { cava } \\
\text { simulado }\end{array}$ & $\begin{array}{c}\text { TBS } \\
\text { interior } \\
\text { máxima } \\
\left({ }^{\circ} \mathbf{C}\right)\end{array}$ & $\begin{array}{c}\text { TBS } \\
\text { interior } \\
\text { mínima } \\
\left({ }^{\circ} \mathbf{C}\right)\end{array}$ & $\begin{array}{c}\text { TBS } \\
\text { interior } \\
\text { promedio } \\
\left({ }^{\circ} \mathbf{C}\right)\end{array}$ & $\begin{array}{c}\text { Oscilación } \\
\text { entre TBS } \\
\text { máxima y } \\
\text { mínima } \\
\left({ }^{\circ} \mathbf{C}\right)\end{array}$ & $\begin{array}{c}\text { Horas } \\
\text { fuera } \\
\text { del } \\
\text { rango }\end{array}$ \\
$\begin{array}{l}\text { optimo } \\
(\%)\end{array}$ \\
\hline $\begin{array}{l}\text { Edificio } \\
\text { Base }\end{array}$ & 19.31 & 13.24 & 15.62 & 6.07 & 62.77 \\
\hline $\begin{array}{l}\text { Edificio } \\
\text { semi- } \\
\text { enterrado }\end{array}$ & 19.12 & 13.42 & 15.70 & 5.70 & 69.09 \\
\hline $\begin{array}{l}\text { Edificio } \\
\text { subterráneo }\end{array}$ & 18.46 & 13.35 & 15.44 & 5.11 & 53.16 \\
\hline $\begin{array}{l}\text { Edificio } \\
\text { subterráneo } \\
\text { con techo } \\
\text { verde }\end{array}$ & 18.01 & 14.40 & 16.11 & 3.61 & 87.73 \\
\hline $\begin{array}{l}\text { Edificio } \\
\text { subterráneo } \\
\text { con doble } \\
\text { techo }\end{array}$ & 18.23 & 12.98 & 15.13 & 5.25 & 21.64 \\
\hline $\begin{array}{l}\text { Edificio } \\
\text { subterráneo } \\
\text { con } \\
\text { poliuretano } \\
0.10 \text { m. }\end{array}$ & 18.21 & 14.05 & 15.82 & & \\
\hline
\end{tabular}

Tabla 3 Resultados de temperatura interior en modelos de cava de vino. Periodo frío área de envejecimiento

Fuente: Elaboración propia

2) Los datos horarios obtenidos de temperatura de bulbo seco interior permitieron realizar un análisis mensual de los grados-hora del área de envejecimiento. En el cálculo se consideró el rango de temperatura establecido para dicha área de $10{ }^{\circ} \mathrm{C}$ a $15{ }^{\circ} \mathrm{C}$. La fórmula condicional utilizada fue la siguiente:

Si $\mathrm{X}>15$ entonces $\mathrm{Gh}=\mathrm{X}-15$

Si $\mathrm{X}<15 \leq 10$ entonces $\mathrm{Gh}=0$

Donde Gh son los grados-hora y X es la temperatura de bulbo seco horario interior obtenida con la simulación térmica.

La cantidad mínima de grados-hora requeridos se presentó en el edificio con la estrategia del techo doble seguido del modelo con poliuretano, el edificio con muros subterráneos resultó ser la mejor configuración seguido del modelo con muros semi-enterrados (Figura 9).

El mes con mayor cantidad de gradoshora por encima del rango fue diciembre y el mes con menor cantidad de grados-hora fue febrero. Lo anterior mostró que en este periodo los edificios tienden a almacenar calor, aunado a la carga térmica aportada por los procesos del vino dentro de las barricas y actividades operativas del área. 


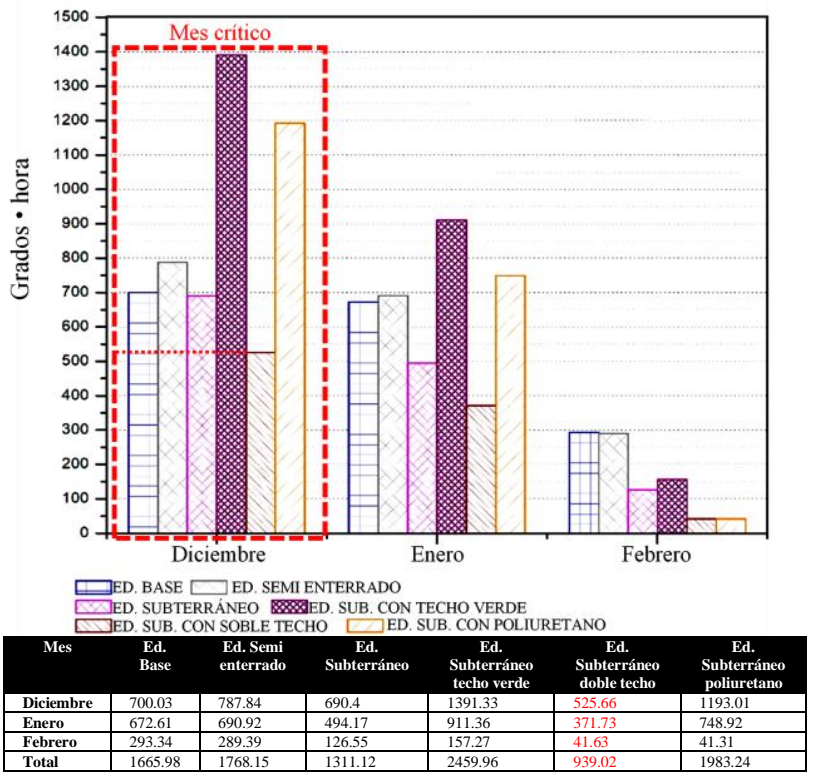

Figura 9 Grados-hora mensuales del área de envejecimiento, periodo frío

Fuente: Elaboración propia

3) El análisis de requerimiento de enfriamiento sensible por zona $(\mathrm{kWh})$ mostró que los modelos con menor requerimiento de enfriamiento fueron el edificio con techo doble, el edificio con muros subterráneos y el edificio con aislamiento térmico con poliuretano (Figura 10). Es importante mencionar que en estos gráficos los valores de $\mathrm{kWh}$ se muestran en negativo debido a que es la cantidad de energía que se requiere retirar del edificio.

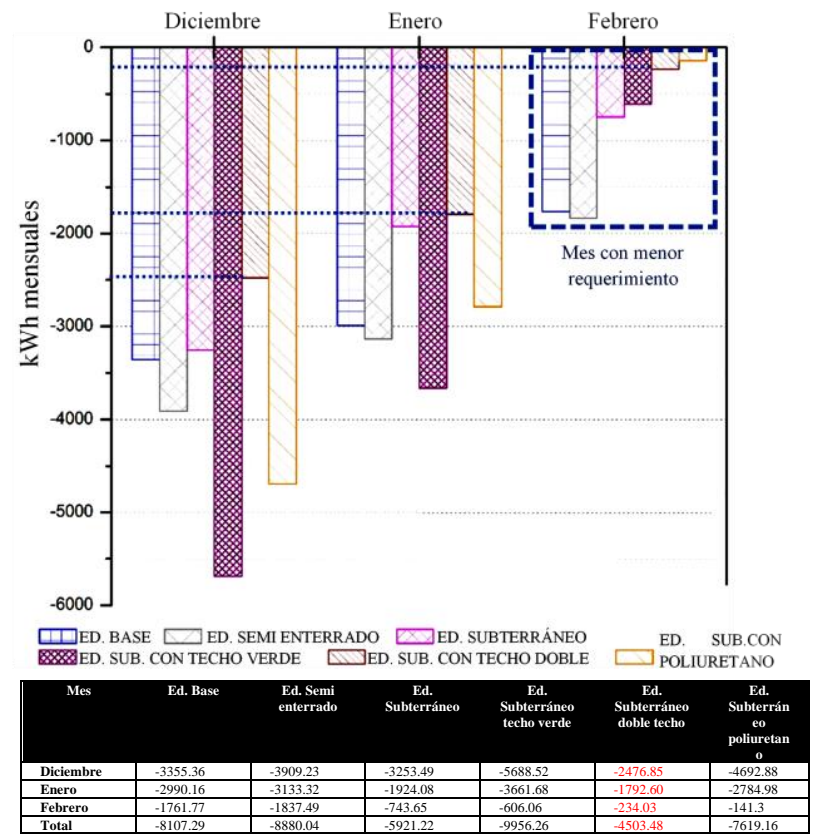

Figura 10 Enfriamiento sensible. Área de envejecimiento periodo frío

Fuente: Elaboración propia
Aunque la estrategia del techo doble presentó menor cantidad de $\mathrm{kWh}$ mensuales en comparación con el techo verde, este último tuvo menor oscilación en la temperatura interior, lo que significó mayor estabilidad de las condiciones ambientales para el vino. En febrero, el mes con menor requerimiento de enfriamiento sensible, el edificio con techo con poliuretano requirió menor cantidad de $\mathrm{kWh}$ para mantener el área de envejecimiento dentro del rango recomendado de temperatura interior, en comparación con los demás edificios.

Al comparar los totales del periodo, por edificio simulado, se obtuvo que el mayor requerimiento de enfriamiento fue el del edificio subterráneo con techo verde con 9,956 kWh, lo cual indicó que la masa térmica en contacto con la cubierta permite almacenar el calor y retrasa las pérdidas de energía de la edificación, mientras que la menor cantidad de enfriamiento sensible requerido se logró en el edificio subterráneo con cubierta doble con 4,503 kWh, esto debido a que la segunda cubierta proporcionó sombreado al edificio en horas de mayor radiación solar y permitió el contacto con el aire del exterior, esta estrategia disminuyó la ganancia de energía por techo $45 \%$ menos en comparación con el edificio base, que se consideró como el caso crítico sin estrategias aplicadas (Figura 9).

\section{Periodo cálido}

Incluyó los meses de mayo a septiembre, se analizaron: 1) Temperatura de bulbo seco interior $\left.\left({ }^{\circ} \mathrm{C}\right), 2\right)$ Grados-hora mensuales $\left({ }^{\circ} \mathrm{C}\right) 3$ ) Enfriamiento o calentamiento sensible (kWh). El 99\% de las horas del periodo cálido la TBS se ubicó fuera del rango óptimo establecido de 10 ${ }^{\circ} \mathrm{C}$ a $15^{\circ} \mathrm{C}$, el resto de las horas se ubicaron dentro del rango. Lo anterior permitió definir que para esta área es necesario el uso de sistemas electromecánicos de refrigeración la mayor parte de las horas del periodo.

1) Los resultados obtenidos de temperatura de bulbo seco (TBS) interior, mostraron que en el edificio base la TBS máxima interior fue de $24.87^{\circ} \mathrm{C}$, en el edificio semienterrado de $24.57^{\circ} \mathrm{C}$, en el edificio subterráneo $23.23{ }^{\circ} \mathrm{C}$, en el edificio subterráneo con techo verde $21.23^{\circ} \mathrm{C}$, en el modelo con doble techo $21.43{ }^{\circ} \mathrm{C}$ y en el edificio con poliuretano en la cubierta $20.59{ }^{\circ} \mathrm{C}$. 
El mejor desempeño en cuanto a TBS interior se obtuvo con el modelo con aislante térmico de poliuretano, una diferencia de $17 \%$ menos en comparación con el edificio base considerado como caso crítico, y $3 \%$ menor en comparación con el modelo con techo verde (Tabla 4).

\begin{tabular}{|l|c|r|r|r|r|}
\hline $\begin{array}{l}\text { Modelo de } \\
\text { cava } \\
\text { simulado }\end{array}$ & $\begin{array}{c}\text { TBS } \\
\text { interior } \\
\text { máxima } \\
\left({ }^{\circ} \mathbf{C}\right)\end{array}$ & $\begin{array}{c}\text { TBS } \\
\text { interior } \\
\text { mínima } \\
\left({ }^{\circ} \mathbf{C}\right)\end{array}$ & $\begin{array}{c}\text { TBS } \\
\text { interior } \\
\text { promedio } \\
\left({ }^{\circ} \mathbf{C}\right)\end{array}$ & $\begin{array}{c}\text { Oscilación } \\
\text { entre TBS } \\
\text { máxima y } \\
\text { mínima } \\
\left({ }^{\circ} \mathbf{C}\right)\end{array}$ & $\begin{array}{c}\text { Horas } \\
\text { fuera } \\
\text { del } \\
\text { rango }\end{array}$ \\
$\begin{array}{l}\text { optimo } \\
(\%)\end{array}$ \\
\hline $\begin{array}{l}\text { Edificio } \\
\text { Base }\end{array}$ & 24.87 & 15.10 & 20.83 & 9.77 & 100.00 \\
\hline $\begin{array}{l}\text { Edificio } \\
\text { semi- } \\
\text { enterrado }\end{array}$ & 24.57 & 15.14 & 20.69 & 9.43 & 100.00 \\
\hline $\begin{array}{l}\text { Edificio } \\
\text { subterráneo }\end{array}$ & 23.23 & 14.83 & 19.86 & 8.40 & 99.75 \\
\hline $\begin{array}{l}\text { Edificio } \\
\text { subterráneo } \\
\text { con techo } \\
\text { verde }\end{array}$ & 21.23 & 15.08 & 18.82 & 6.15 & 100.00 \\
\hline $\begin{array}{l}\text { Edificio } \\
\text { subterráneo } \\
\text { con doble } \\
\text { techo }\end{array}$ & 21.43 & 14.00 & 18.48 & 7.43 & 99.42 \\
\hline $\begin{array}{l}\text { Edificio } \\
\text { subterráneo } \\
\text { con } \\
\text { poliuretano } \\
0.10 \mathrm{~m}\end{array}$ & 20.59 & 14.59 & 18.23 & 5.99 & 99.75 \\
\hline
\end{tabular}

Tabla 4 Resultados de temperatura interior en modelos de cava de vino. Periodo cálido área de envejecimiento Fuente: Elaboración propia

2) En el análisis de grados-hora para el área de envejecimiento se obtuvo que los meses críticos del periodo cálido fueron julio, agosto y septiembre. Los modelos con menor cantidad de grados hora mensuales fueron el edificio con poliuretano, el edificio con doble techo y el edificio con techo verde (Figura 11).

En el mes de agosto (cuando se registraron los valores máximos) el edificio base tuvo 5,443 grados-hora por encima de $\operatorname{los} 15^{\circ} \mathrm{C}$, que fue el límite máximo del rango óptimo, mientras que en el edificio con poliuretano se estimaron 3,333 grados-hora, 38\% menos y en el edificio con techo verde durante el mismo mes se observaron 3,823 grados-hora, una diferencia en comparación con el edificio base de $30 \%$ y del $13 \%$ en comparación con el edificio con poliuretano (Figura 11).

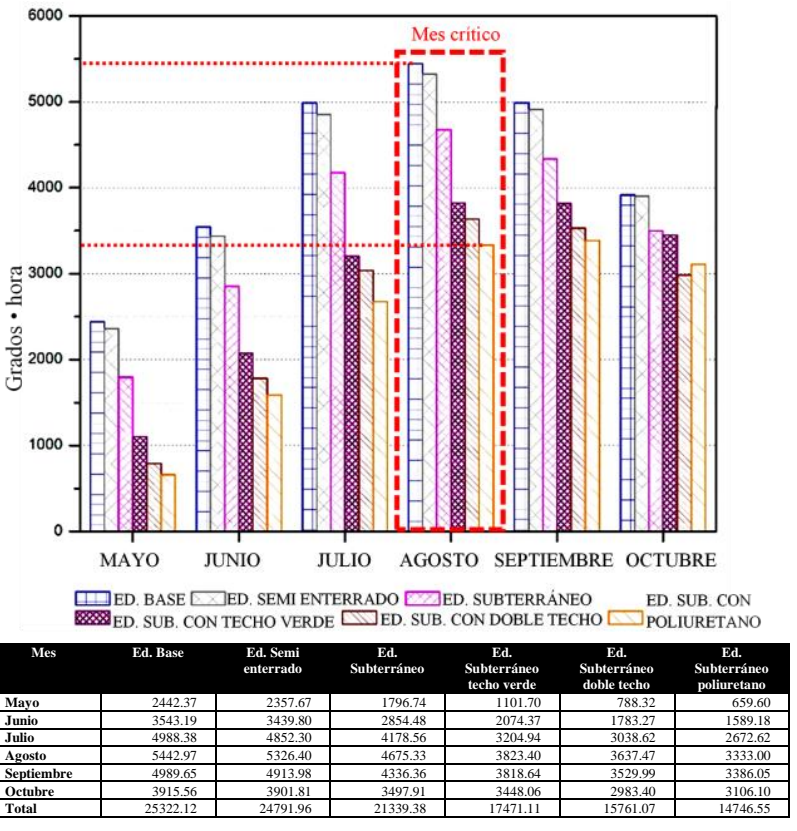

Figura 11 Grados-hora mensuales del área de envejecimiento, periodo cálido

Fuente: Elaboración propia

3) El enfriamiento sensible se analizó a partir de $\mathrm{kWh}$, igual que en el análisis de gradoshora agosto fue el mes crítico del periodo, el que requirió mayor cantidad de energía para enfriamiento del área de envejecimiento (Figura 12).
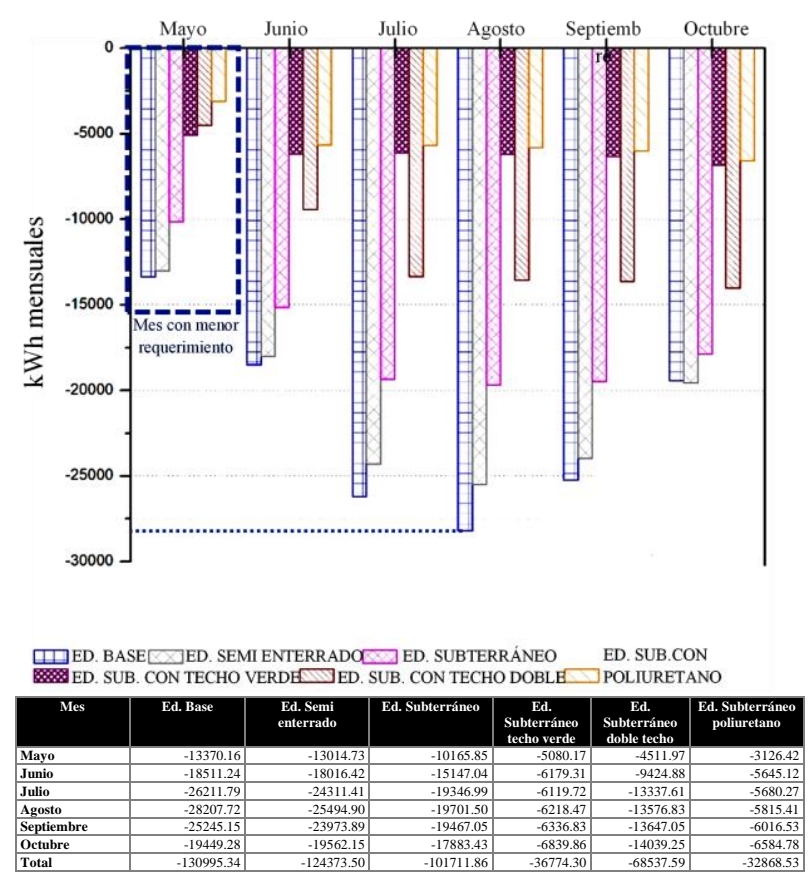

Figura 12 Enfriamiento sensible (kWh). Área de envejecimiento periodo cálido

Fuente: Elaboración propia 
El requerimiento de enfriamiento mensual en agosto fue de más de 28,207 kWh para el edificio base, mientras que para el edificio con poliuretano el enfriamiento total para el mismo mes fue de $5,815 \mathrm{kWh}$, una diferencia de $80 \%$. El modelo que presentó un buen desempeño fue el edificio con techo verde con una diferencia de $78 \%$ menos $\mathrm{kWh}$ en comparación con el edificio base y de $6.5 \%$ en comparación con el modelo con aislamiento térmico de poliuretano. Estos resultados indican que los techos verdes son una estrategia bioclimática adecuada para cumplir con los requerimientos de temperatura y humedad interior del área de envejecimiento en edificios vinícolas.

Es importante mencionar que todos los meses del año se requirió de enfriamiento, principalmente en los meses de junio a octubre, incluso en los meses del periodo frío, esto puede deberse al rango de temperatura utilizado para las simulaciones del área de envejecimiento, que fue de $10^{\circ} \mathrm{C}$ a $15^{\circ} \mathrm{C}$ (Tabla 1 ).

\section{Conclusiones}

Las estrategias bioclimáticas de masa térmica, sombreado y enfriamiento electromecánico aplicadas en el diseño de edificios vinícolas artesanal del Valle de Guadalupe, optimizan el desempeño higrotérmico del edificio al generar un ambiente interior dentro de los rangos de temperatura de bulbo seco y humedad relativa adecuados para el envejecimiento del vino. Un correcto diseño bioclimático de este tipo de edificios que requieren un control estricto de temperatura y humedad interior puede ser una alternativa sustentable para minimizar el consumo energético por climatización, tanto de construcciones nuevas, así como de recuperaciones o remodelaciones.

La ubicación constructiva del área de envejecimiento del vino resultó ser determinante en la oscilación de la temperatura ambiente interior, este efecto térmico coincide con lo que sucede en las cavas subterráneas tradicionales españolas que aún se utilizan. El modelo subterráneo combinado con un techo verde permitió disminuir la temperatura interior en los meses del periodo cálido, su desempeño fue parecido al obtenido con $0.10 \mathrm{~m}$ de aislamiento con poliuretano, considerado como el sistema más utilizado en la región y que tiene menos ventajas ambientales que un techo verde.
En el periodo frío, el modelo con mejor desempeño térmico fue el del techo verde, debido al efecto de almacenamiento de calor por la masa térmica en contacto directo con la cubierta, se obtuvieron valores por encima del rango óptimo definido, sin embargo, su diferencial de temperatura entre máxima y mínima fue de $3.61{ }^{\circ} \mathrm{C}$, la menor en comparación con los otros modelos. En el periodo cálido también tuvo el menor diferencial de TBS, con $6.15^{\circ} \mathrm{C}$. En los dos casos se tuvieron valores muy cercanos a los obtenidos con el modelo con poliuretano, con menos del $35 \%$ de diferencia.

La estrategia bioclimática que se recomienda para asegurar un óptimo desempeño higrotérmico en el envejecimiento del vino fue el techo verde, además de que se recupera el suelo utilizado al restituir con áreas verde el suelo ocupado por el edificio, puede ser atractivo para el turismo y servir como huerto urbano o si su diseño lo permite puede incluir la plantación de variaciones de vid.

El edificio con doble cubierta también resultó ser una estrategia bioclimática que redujo la temperatura de bulbo seco interior, su costo por construcción puede ser menor en comparación con un techo verde e igual de atractivo cuando se considera su diseño, además de que puede ser un elemento de adecuación para edificios ya existentes.

Puede concluirse que el ambiente térmico interior de un edificio ubicado en el Valle de Guadalupe sin ninguna estrategia aplicada ya sea pasivo o activa, se encontrará arriba del rango óptimo para envejecimiento del vino $\left(10^{\circ} \mathrm{C}\right.$ a $\left.15^{\circ} \mathrm{C}\right)$ el $100 \%$ de las horas del periodo cálido y más del $60 \%$ del periodo frío, lo que requerirá el uso obligado de sistemas electromecánicos de enfriamiento para asegurar la calidad del producto y evitar pérdidas de vino por evaporación. No considerar el diseño bioclimático de los edificios vinícolas puede ser una amenaza a futuro para la calidad del vino producido en la zona, debido a la poca preparación de los edificios para contrarrestar los incrementos de temperatura por cambio climático. 


\section{Agradecimientos}

Agradezco la cooperación e interés del Dr. Víctor Torres y el Ing. Aime Desponds para la realización de este trabajo, propietarios de vinícolas de la zona de estudio. Al Dr. Adolfo Gómez Amador, por su contribución académica en la revisión del trabajo de investigación. A Luca Bojórquez y Zuly Bojórquez. A la Facultad de Arquitectura y Diseño y al Instituto de Ingeniería de la Universidad Autónoma de Baja California. Al Consejo Nacional de Ciencia y Tecnología por la beca otorgada para realizar estudios de doctorado.

\section{Referencias}

Ali, M. H. y Abustan, I. (2014). A new novel index for evaluating model performance. Journal of natural resources and development, 04: 1-9. DOI: 10.5027/jnrd.v4i0.01.

Barbaresi, A., Torregiani, D., Benni, S. y Tassinari, P. (2014). Underground cellar thermal simulation: Definition of a method for modelling performance assessment based on experimental calibration. Energy and Buildings, 76: 363-372.

Boulton, R. B., Singleton, V. L., Bisson, L. F. y Kunkee, R. E. (1995). Teoría y práctica de la elaboración del vino. Editorial: Acribia S. A. Zaragoza, España. Págs. 636.

Cavazos, M.T. (2012). Situación actual y bajo escenarios de cambio climático de la industria vitivinícola de Baja California, México. Departamento de Oceanografía Física, CICESE. Baja California. Págs. 76.

Considine, J. A. y Frankish, E. (2014). A complete guide to quality in small-scale wine making. Págs. 224. ISBN: 9780124080812.

Hidalgo, J. (2003). Tratado de Enología (Treatise of Enology), Ediciones Mundi - Prensa, Madrid, España.

Marrara, V., Barreca, F. y Di Fazio, S. (2014). Green roofs in the sustainable design of agrifood build-ings: a case-study in Calabria (Italy). Proceedings International Conference of Agricultural Engineering, Zurich. Págs. 8.
Mateus, N., Pinto, A. y Carrilho da Graca, G. (2014). Validation of EnergyPlus thermal simulation of a double skin naturally and mechanically ventilated test cell. Energy and Buildings, 75: 511 - 522.

Mazarrón, F. y Cañas, I. (2008). Exponential sinusoidal model for predicting temperature inside underground wine cellars from a Spanish region. Energy and Buildings, 40: 1931 - 1940.

Mazarrón, F., Cid - Falceto, J. y Cañas, I. (2012). An assessment of using ground thermal inertia as passive thermal technique in the wine industry around the world. Applied Thermal Engineering, 33-34: 54-61.

Sailor, D. J. (2008). A green roof model for building energy simulation programs. Energy and Buildings, 40: 1466-1478.

San Isidro, J. (1998). Proceso de validación de modelos de simulación. Informes técnicos del Centro de Investigaciones Energéticas, Medioambientales y Tecnológicas (CIEMAT). ISSN: 1135-9420.

SEPSA. (1952). Guía práctica de vinificación moderna. 4th ed. Barcelona, España: Ediciones Sociedad Enológica del Penedés.

Steiner, T. E. (2010). What is the best sterilization option for the bottling line? Winemaking problems solved. Woodhead Publishing Series. Food Science,Technology and Nutrition, 193. Págs. 150 - 153.

Tassinari, P., Torregiani, D., Benni, S. y Corzani, V. (2012). Research of dimensioning criteria for farm winery design: análisis of spatial layouts in a study area. Structures and Environmental Technologies. International Conference of Agricultural Engineering - CIGRAgEng 2012: agriculture and engineering for a healthier life, Valencia, España.

Torregiani, D., Benni, S., Corzani, V., Tassinari, P. y Galassi, S. (2011). A meta-design approach to agroindustrial buildings: A case study for typical italian wine productions. Land Use Policy, 28: 11-18.

Troost G. (1953). Die Technologie des Weines. Stuttgart: Eugen Ulmer. 
Ziogou, I., Apostolos, M., Vasiliki, V. y Theodoros, Z. (2017). Energy, environmental and economic assessment of electricity savings from the operation of green roofs in urban office buildings of a warm Mediterranean region. Journal of Cleaner Production, 168: 346-356. 